Bull. Korean Math. Soc. 48 (2011), No. 6, pp. 1253-1259

http://dx.doi.org/10.4134/BKMS.2011.48.6.1253

\title{
GENERALIZED DERIVATIONS ON SEMIPRIME RINGS
}

\author{
Vincenzo De Filippis and Shuliang Huang
}

\begin{abstract}
Let $R$ be a prime ring, $I$ a nonzero ideal of $R$ and $n$ a fixed positive integer. If $R$ admits a generalized derivation $F$ associated with a derivation $d$ such that $(F([x, y]))^{n}=[x, y]$ for all $x, y \in I$. Then either $R$ is commutative or $n=1, d=0$ and $F$ is the identity map on $R$. Moreover in case $R$ is a semiprime ring and $(F([x, y]))^{n}=[x, y]$ for all $x, y \in R$, then either $R$ is commutative or $n=1, d(R) \subseteq Z(R), R$ contains a non-zero central ideal and $F(x)-x \in Z(R)$ for all $x \in R$.
\end{abstract}

\section{Introduction}

Let $R$ be a prime ring with center $Z(R)$ and extended centroid $C, U$ the Utumi quotients ring (for more details on these objects we refer the reader to $[3])$. We denote by $[a, b]=a b-b a$ the simple commutator of the elements $a, b \in R$ and by $a \circ b=a b+b a$ the simple anti-commutator of $a, b$. Recall that a ring $R$ is prime if for any $a, b \in R, a R b=(0)$ implies either $a=0$ or $b=0$, and it is semiprime if for any $a \in R, a R a=(0)$ implies $a=0$. Let $f: R \rightarrow R$ be an additive mapping on $R$. It is a derivation if $f(x y)=f(x) y+x f(y)$ holds for all $x, y \in R$. It is a left multiplier if $f(x y)=f(x) y$ for all $x, y \in R$.

An additive mapping $F: R \rightarrow R$ is called a generalized derivation if there exists a derivation $d: R \rightarrow R$ such that $F(x y)=F(x) y+x d(y)$ holds for all $x, y \in R$, and $d$ is called the associated derivation of $F$. Hence, the concept of generalized derivations covers both the concepts of derivations and left multipliers. Basic examples of generalized derivations are mappings of type $x \rightarrow a x+x b$ for some $a, b \in R$. These maps are called inner generalized derivations. More informations on generalized derivations can be found in [8]. We would like to point out that in [11] Lee proved that every generalized derivation can be uniquely extended to a generalized derivation of $U$ and thus all generalized derivations of $R$ will be implicitly assumed to be defined on the whole $U$. In particular Lee proves the following result:

Received July 16, 2010; Revised April 20, 2011.

2010 Mathematics Subject Classification. 16N60, 16W25.

Key words and phrases. prime and semiprime rings, differential identities, generalized derivations.

The second author was supported by the Natural Science Research Foundation of Anhui Provincial Education Department(No. KJ2010B144).

(C)2011 The Korean Mathematical Society 
Fact 1 ([11, Theorem 4]). Let $R$ be a semiprime ring. Then every generalized derivation $g$ on a dense right ideal of $R$ can be uniquely extended to $U$ and assumes the form $g(x)=a x+d(x)$ for some $a \in U$ and a derivation $d$ on $U$.

In [5, Theorem 2], Daif and Bell showed that if $R$ is a semiprime ring, $I$ is a nonzero ideal of $R$ and $d: R \rightarrow R$ is a derivation such that $d([x, y])=[x, y]$ for all $x, y \in I$, then $I \subseteq Z(R)$.

Later in [14], Quadri et al. discussed the commutativity of prime rings with generalized derivations. More precisely, they proved that if $R$ is a prime ring, $I$ a nonzero ideal of $R$ and $F$ a generalized derivation associated with a nonzero derivation $d$ such that $F([x, y])=[x, y]$ for all $x, y \in I$, then $R$ is commutative.

In [2, Theorem 4.1], Ashraf and Rehman obtained a similar result in case $R$ is a prime ring, replacing the simple commutator by the simple anti-commutator. They proved that if $I$ is a nonzero ideal of $R$ and $d$ is a derivation of $R$ such that $d(x \circ y)=x \circ y$ for all $x, y \in I$, then $R$ is commutative.

More recently in [1, Theorem 1], Argac and Inceboz generalized the above result as follows: Let $R$ be a prime ring, $I$ a nonzero ideal of $R$ and $n$ a fixed positive integer; if $R$ admits a derivation $d$ with the property $(d(x \circ y))^{n}=x \circ y$ for all $x, y \in I$, then $R$ is commutative.

Motivated by these results, we study prime and semiprime rings admitting a generalized derivation $F$ satisfying a condition $(F([x, y]))^{n}=[x, y]$.

\section{The results}

Firstly we consider the case when $R$ is a prime ring and begin with the following:

Remark 1. If $I$ is a non-zero ideal of the prime ring $R$, then:

(1) $I, R$ and $U$ satisfy the same generalized polynomial identities with coefficients in $U$ (Theorem 2 in [4]);

(2) $I, R$ and $U$ satisfy the same differential identities (Theorem 2 in [12]).

Theorem 1. Let $R$ be a prime ring, $I$ a nonzero ideal of $R$ and $n$ a fixed positive integer. If $R$ admits a generalized derivation $F$ associated with a derivation $d$ such that $(F([x, y]))^{n}=[x, y]$ for all $x, y \in I$, then either $R$ is commutative or $n=1, d=0$ and $F$ is the identity map on $R$.

Proof. Assume first that $n=1$. In view of Theorem 2.1 in [14], we have either $R$ is commutative or $d=0$. Consider now this last case and assume that $R$ is not commutative. Thus $F(x y)=F(x) y$ for all $x, y \in R$. Let $x, y, z \in I$, then $x z \in I$. By the hypothesis it follows $F([x z, y])=[x z, y]$ and expanding this we have $(F(x)-x)[z, y]=0$. Replace now $z$ by $z r \in I$ for any $r \in R$. Thus one has $0=(F(x)-x)[z r, y]=(F(x)-x) z[r, y]$, which means $(F(x)-x) I[R, I]=(0)$ for all $x \in R$. Thus, by the primeness of $R$ and since $R$ is assumed not commutative, it follows that $F(x)=x$ for all $x \in I$. Hence, for any $s \in R$ we have $s x \in I$ and $s x=F(s x)=F(s) x$, i.e., $(s-F(s)) I=0$ which implies $F(s)=s$ for all $s \in R$ and $F$ is the identity map on $R$. 
Assume now that $n \geq 2$. By Fact 1 we have that for all $x \in R, F(x)=$ $a x+d(x)$ for some $a \in U$ and a derivation $d$ on $U$. By the given hypothesis we have now $[x, y]=(a[x, y]+d([x, y]))^{n}=(a[x, y]+[d(x), y]+[x, d(y)])^{n}$ for all $x, y \in I$. This means that $I$ satisfies the generalized differential identity

$$
\left(a\left[x_{1}, x_{2}\right]+\left[d\left(x_{1}\right), x_{2}\right]+\left[x_{1}, d\left(x_{2}\right)\right]\right)^{n}-\left[x_{1}, x_{2}\right] .
$$

Since $I$ and $U$ satisfy the same differential identities (Remark 1) we also have that $U$ satisfies (1). We divide the proof into two cases:

Firstly we assume that $d$ is the inner derivation induced by some element $q \in U$, that is $d(x)=[q, x]$ for all $x, y \in U$. In this case we will prove that $q \in C$.

Notice that $U$ satisfies the generalized polynomial identity

$$
\left(a\left[x_{1}, x_{2}\right]+\left[\left[q, x_{1}\right], x_{2}\right]+\left[x_{1},\left[q, x_{2}\right]\right]\right)^{n}-\left[x_{1}, x_{2}\right] .
$$

In case the center $C$ of $U$ is infinite, we have that $\left(a\left[x_{1}, x_{2}\right]+\left[\left[q, x_{1}\right], x_{2}\right]+\right.$ $\left.\left[x_{1},\left[q, x_{2}\right]\right]\right)^{n}-\left[x_{1}, x_{2}\right]$ is a generalized polynomial identity for $U \otimes_{C} \bar{C}$, where $\bar{C}$ is the algebraic closure of $C$. Remark that, in light of Remark $1,[q, x]$ is a generalized polynomial identity for $U$ if and only if it is a generalized identity also for $R$; analogously $U$ is commutative if and only if $R$ is commutative. Therefore, in order to prove that either $q \in C$ or $R$ is commutative, we may replace $R$ by $U$ or $U \otimes_{C} \bar{C}$ according as $C$ is finite or infinite. Moreover, since both $U$ and $U \otimes_{C} \bar{C}$ are prime and centrally closed (Theorem 2.5 and Theorem 3.5 in [6]), we may assume that $R$ is centrally closed over $C$ (i.e., $R C=C$ ) which is either finite or algebraically closed and $(a[x, y]+[[q, x], y]+[x,[q, y]])^{n}=[x, y]$ for all $x, y \in R$. By Theorem 3 in [13], $R$ is a primitive ring which is isomorphic to a dense ring of linear transformations of a vector space $V$ over a division ring $D$.

Assume that $\operatorname{dim} V_{D} \geq 3$. Our first aim is to show that $v$ and $q v$ are linearly $D$-dependent for all $v \in V$. Suppose there exists $v \in V$ such that $v$ and $q v$ are $D$-independent. Since $\operatorname{dim} V_{D} \geq 3$, then there exists $w \in V$ such that $v, q v, w$ are also $D$-independent. By the density of $R$, there exist $x, y \in R$ such that: $x v=0, x q v=w, x w=v ; y v=0, y q v=0, y w=v$. These imply that $v=(a[x, y]+[[q, x], y]+[x,[q, y]])^{n} v=[x, y] v=x y v-y x v=0$, which is a contradiction. So we conclude that $v$ and $q v$ are linearly $D$-dependent for all $v \in V$

Our next goal is to show that there exists $b \in D$ such that $q v=v b$ for all $v \in V$. In fact, choose $v, w \in V$ linearly $D$-independent. Since $\operatorname{dim} V_{D} \geq 3$, then there exists $u \in V$ such that $u, v, w$ are linearly $D$-independent, and so $b_{u}, b_{v}, b_{w} \in D$ such that $q u=u b_{u}, q v=v b_{v}, q w=w b_{w}$, that is $q(u+v+w)=$ $u b_{u}+v b_{v}+w b_{w}$. Moreover $q(u+v+w)=(u+v+w) b_{u+v+w}$ for a suitable $b_{u+v+w} \in D$. Then $0=u\left(b_{u+v+w}-b_{u}\right)+v\left(b_{u+v+w}-b_{v}\right)+w\left(b_{u+v+w}-b_{w}\right)$ and because $u, v, w$ are linearly $D$-independent, $b_{u}=b_{v}=b_{w}=b_{u+v+w}$, that is $b$ does not depend on the choice of $v$. Hence now we have $q v=v b$ for all $v \in V$. 
Now for $r \in R, v \in V$, we have $(r q) v=r(q v)=r(v b)=(r v) b=q(r v)$, that is $[q, R] V=0$. Since $V$ is a left faithful irreducible $R$-module, hence $[q, R]=0$, i.e., $q \in C$ and so $d=0$.

Therefore $(a[x, y])^{n}=[x, y]$ for all $x, y \in R$. Suppose there exists $v \in V$ such that $v$ and $a v$ are $D$-independent. Since $\operatorname{dim} V_{D} \geq 3$, there exists $w \in V$ such that $v, a v, w$ are also $D$-independent. By the density of $R$, there exist $x, y \in R$ such that $x v=0, y v=w, x w=v, x a v=0, y a v=0$. Hence it follows the contradiction $v=[x, y] v=(a[x, y])^{n} v=0$. Therefore, using the same above argument, we have that $a \in C$. This means that $a^{n}[x, y]^{n}=[x, y]$ for all $x, y \in R$. Again fix $v_{1}, v_{2}, v_{3} \in V$ linearly $D$-independent vectors. As above there exist $x, y \in R$ such that $x v_{1}=0, y v_{1}=v_{2}, x v_{2}=v_{3}, x v_{3}=0, y v_{3}=0$. Finally we have the contradiction $v_{3}=[x, y] v_{1}=a^{n}[x, y]^{n} v_{1}=0$.

Suppose now that $\operatorname{dim} V_{D} \leq 2$. In this case $R$ is a simple GPI-ring with 1 , and so it is a central simple algebra finite dimensional over its center. By [10] (Lemma 2), it follows that there exists a suitable field $E$ such that $R \subseteq$ $M_{k}(E)$, the ring of all $k \times k$ matrices over $E$, and moreover $M_{k}(E)$ satisfies the same generalized polynomial identities of $R$. In particular $M_{k}(E)$ satisfies $\left(a\left[x_{1}, x_{2}\right]+\left[\left[q, x_{1}\right], x_{2}\right]+\left[x_{1},\left[q, x_{2}\right]\right]\right)^{n}-\left[x_{1}, x_{2}\right]$. If $k \geq 3$, by the same above argument we get $q \in C$. Obviously if $k=1$, then $R$ is commutative.

Thus we may assume that $k=2$, i.e., $R \subseteq M_{2}(E)$, where $M_{2}(E)$ satisfies $\left(a\left[x_{1}, x_{2}\right]+\left[\left[q, x_{1}\right], x_{2}\right]+\left[x_{1},\left[q, x_{2}\right]\right]\right)^{n}-\left[x_{1}, x_{2}\right]$.

Denote $e_{i j}$ the usual matrix unit with 1 in $(i, j)$-entry and zero elsewhere. Let $\left[x_{1}, x_{2}\right]=\left[e_{21}, e_{11}\right]=e_{21}$. In this case we have $\left(a e_{21}+q e_{21}-e_{21} q\right)^{n}=e_{21}$. Right multiplying by $e_{21}$, we get

$$
(-1)^{n}\left(e_{21} q\right)^{n} e_{21}=\left(a e_{21}+q e_{21}-e_{21} q\right)^{n} e_{21}=e_{21} e_{21}=0 .
$$

Set $q=\sum_{i, j=1}^{2} q_{i j} e_{i j}$, with $q_{i j} \in E$. By calculation we find that $(-1)^{n} q_{12}^{n} e_{21}$ $=0$, which implies that $q_{12}=0$. Similarly we can see that $q_{21}=0$. Therefore $q$ is diagonal in $M_{2}(E)$. Let $f$ be any automorphism of $M_{2}(E)$ and notice that $(f(a)[f(x), f(y)]+[[f(q), f(x)], f(y)]+[f(x),[f(q), f(y)]])^{n}=[f(x), f(y)]$. Thus the same above argument shows that $f(q)$ is a diagonal matrix in $M_{2}(E)$. In particular, let $f(x)=\left(1-e_{i j}\right) x\left(1+e_{i j}\right)$ for $i \neq j$, then $f(q)=q+\left(q_{i i}-q_{j j}\right) e_{i j}$, that is $q_{i i}=q_{j j}$ for $i \neq j$. This implies again that $q$ is central in $M_{2}(E)$. Therefore $d=0$ and $M_{2}(E)$ satisfies the generalized identity $\left(a\left[x_{1}, x_{2}\right]\right)^{n}-$ $\left[x_{1}, x_{2}\right]$. Let $\left[x_{1}, x_{2}\right]=e_{21}$. Thus we have $\left(a e_{21}\right)^{n}=e_{21}$. Analogously, for $\left[x_{1}, x_{2}\right]=e_{12}$ we have that $\left(a e_{12}\right)^{n}=e_{12}$. As above we obtain that $a$ is a diagonal matrix and using the same above argument, we conclude that $a$ is a central matrix. Thus $M_{2}(E)$ satisfies $a^{n}\left[x_{1}, x_{2}\right]^{n}-\left[x_{1}, x_{2}\right]$. In this case we notice that, for $\left[x_{1}, x_{2}\right]=e_{12}$, the contradiction $0=e_{12}$ follows.

Assume now that $d$ is not an inner derivation of $U$. Hence, by (1) and the Kharchenko's result in [9], it follows that $U$ satisfies the generalized polynomial identity

$$
\left(a\left[x_{1}, x_{2}\right]+\left[y_{1}, x_{2}\right]+\left[x_{1}, y_{2}\right]\right)^{n}-\left[x_{1}, x_{2}\right] .
$$


As above, we may replace $R$ by $U$ or $U \otimes_{C} \bar{C}$ according as $C$ is finite or infinite, and assume that $R$ is centrally closed over $C$. Thus $R$ satisfies $(2)$ and in particular, $R$ satisfies the blended component $\left(\left[x_{1}, y_{2}\right]\right)^{n}$, that is, $R$ is a ring satisfying a polynomial identity. Hence there exists a suitable field $E$ such that $R \subseteq M_{k}(E)$, the ring of all $k \times k$ matrices over $E$, and moreover $M_{k}(E)$ satisfies the same polynomial identities of $R$. In particular $M_{k}(E)$ satisfies $\left[x_{1}, x_{2}\right]^{n}$. If $k \geq 2$, for $x_{1}=e_{12}$ and $x_{2}=e_{21}$, we get the contradiction $\left(e_{11}-e_{22}\right)^{n}=0$. Thus $k=1$ and then $R$ is commutative.

The following example shows that $R$ to be prime is essential in the hypothesis.

Example. Let $S$ be any ring and let $R=\left\{\left(\begin{array}{ll}a & b \\ 0 & 0\end{array}\right) \mid a, b \in S\right\}$ and let $I=$ $\left\{\left(\begin{array}{ll}0 & a \\ 0 & 0\end{array}\right) \mid a \in S\right\}$ be a nonzero ideal of $R$. We define a map $F: R \rightarrow R$ by $F(x)=2 e_{11} x-x e_{11}$. Then it is easy to see that $F$ is a generalized derivation associated with a nonzero derivation $d(x)=\left[e_{11}, x\right]$. It is straightforward to check that $F$ satisfies the property: $(F([x, y]))^{n}=[x, y]$ for all $x, y \in I$. However, $R$ is not commutative.

Finally we extend the above result to semiprime rings:

Theorem 2. Let $R$ be a semiprime ring and $n$ a fixed positive integer. If $R$ admits a generalized derivation $F$ associated with a derivation $d$ such that $\left(F([x, y])^{n}=[x, y]\right.$ for all $x, y \in R$, then either $R$ is commutative or $n=1$, $d(R) \subseteq Z(R), R$ contains a non-zero central ideal and $F(x)-x \in Z(R)$ for all $x \in R$.

Proof. First consider $n=1$. Let $P$ be a prime ideal of $R$ such that $[R, R] \nsubseteq P$ and set $\bar{R}=R / P$. Assume first that $d(P) \nsubseteq P$. Let $p$ be any element of $P$. Since for all $y \in R, a[p, y]+[d(p), y]+[p, d(y)]=[p, y] \in P$, then $[d(p), y] \in$ $P$, that is $[d(P), R] \subseteq P$. Thus $[d(P R), R] \subseteq P$ and by calculations we get $d(P)[R, R] \subseteq P$. So $d(P)\left[R^{2}, R\right] \subseteq P$ which implies that $d(P) R[R, R] \subseteq P$. By the primeness of $P$ and since $d(P) \nsubseteq P$, it follows that $[R, R] \subseteq P$, a contradiction.

Hence we may assume that $d(P) \subseteq P$, then $d$ induces a canonical derivation $\bar{d}$ on $\bar{R}$. By the assumption we have $\bar{a}[\bar{x}, \bar{y}]+\bar{d}([\bar{x}, \bar{y}])=[\bar{x}, \bar{y}]$ for all $x, y \in R$. It follows from the prime case that one of the following holds:

1. either $[\bar{R}, \bar{R}]=\overline{0}$, that is $[R, R] \subseteq P$, a contradiction; or

2. $\bar{d}(\bar{R})=(\overline{0})$ and $\overline{a x}-\bar{x}=\overline{0}$ for all $x \in R$, that is $d(P) \subseteq P$ and $a x-x \in P$ for all $x \in R$.

In light of previous argument we have that both $d(R)[R, R] \subseteq \bigcap_{i} P_{i}=$ (0) and $(a x-x)[R, R] \subseteq \bigcap_{i} P_{i}=(0)$ for all $x \in R$ (where $P_{i}$ are all prime ideals of $R$ ). Starting from $d(R)[R, R]=0$, we have $0=d\left(R^{2}\right)[d(R), R]=$ $d(R) R[d(R), R]$, in particular we have both $d(R) R^{2}[d(R), R]=0$ and

$$
R d(R) R[d(R), R]=0 .
$$


Therefore $[d(R), R] R[d(R), R]=0$ and by the semiprimeness of $R,[d(R), R]=$ 0 , that is $d(R) \subseteq Z(R)$.

Now consider also $(a x-x)[R, R]=0$. A result of Zalar [15, Lemma 1.3] says that in this case there exists a non-zero central ideal of $R$. Moreover we have that $0=(a x-x)\left[R^{2}, R\right]=(a x-x) R[R, R]$, in particular we have both $(a x-$ $x) R^{2}[R, R]=0$ and $R(a x-x) R[R, R]=0$. Therefore $[a x-x, R] R[R, R]=0$ and a fortiori $[a x-x, R] R[a x-x, R]=0$. By the semiprimeness of $R,[a x-x, R]=0$, that is $a x-x \in Z(R)$ for all $x \in R$. Thus, for all $x \in R$, ax $=x+\alpha_{x}$, where $\alpha_{x} \in Z(R)$ is depending on the choice of $x$; hence $F(x)=a x+\beta_{x}$, where $\beta_{x}=d(x) \in Z(R)$, that is $F(x)=x+\gamma_{x}$ for $\gamma_{x}=\alpha_{x}+\beta_{x} \in Z(R)$.

Let now $n \geq 2$. As above let $P$ be a prime ideal of $R$, and set $\bar{R}=R / P$. Assume first that $d(P) \nsubseteq P$. Let $p$ be any element of $P$. Since for all $y \in$ $R,(a[p, y]+[d(p), y]+[p, d(y)])^{n}-[p, y]=0$, then $[\overline{d(p)}, \bar{y}]=\overline{0}$ in $\bar{R}$ for all $\bar{y} \in \bar{R}$. Since $\bar{R}$ is a prime ring, by a result of Giambruno and Herstein [7] (Theorem 1) either $\bar{R}$ is commutative, that is $[R, R] \subseteq P$, or $\overline{d(p)}$ is central in $\bar{R}$, that is $[d(P), R] \subseteq P$. In this last case, by using the same above argument, one can see that again $[R, R] \subseteq P$. Hence we may assume that $d(P) \subseteq P$, then $d$ induces a canonical derivation $\bar{d}$ on $\bar{R}$. By the assumption we have $(\bar{a}[\bar{x}, \bar{y}]+\bar{d}([\bar{x}, \bar{y}]))^{n}-[\bar{x}, \bar{y}]=\overline{0}$ for all $x, y \in R$. It follows from the prime case that $\bar{R}$ is commutative, that is $[R, R] \subseteq P$. In light of previous argument we have that in any case $[R, R] \subseteq P$. So $[R, R] \subseteq \bigcap_{i} P_{i}=(0)$ (where $P_{i}$ are all prime ideals of $R$ ) and $R$ is commutative.

\section{References}

[1] N. Argac and H. G. Inceboz, Derivations of prime and semiprime rings, J. Korean Math. Soc. 46 (2009), no. 5, 997-1005.

[2] M. Ashraf and N. Rehman, On commutativity of rings with derivations, Results Math. 42 (2002), no. 1-2, 3-8.

[3] K. I. Beidar, W. S. Martindale, and V. Mikhalev, Rings with Generalized Identities, Monographs and Textbooks in Pure and Applied Mathematics, 196. Marcel Dekker, Inc., New York, 1996.

[4] C. L. Chuang, GPI's having coefficients in Utumi quotient rings, Proc. Amer. Math. Soc. 103 (1988), no. 3, 723-728.

[5] M. N. Daif and H. E. Bell, Remarks on derivations on semiprime rings, Internt. J. Math. Math. Sci. 15 (1992), 205-206.

[6] J. S. Erickson, W. S. Martindale III, and J. M. Osborn, Prime nonassociative algebras, Pacific J. Math. 60 (1975), no. 1, 49-63.

[7] A. Giambruno and I. N. Herstein, Derivations with nilpotent values, Rend. Circ. Mat. Palermo (2) 30 (1981), no. 2, 199-206.

[8] B. Hvala, Generalized derivations in rings, Comm. Algebra 26 (1998), no. 4, 1147-1166.

[9] V. K. Kharchenko, Differential identities of prime rings, Algebra and Logic 17 (1978), 155-168.

[10] C. Lanski, An Engel condition with derivation, Proc. Amer. Math. Soc. 118 (1993), no. $3,731-734$.

[11] T. K. Lee, Generalized derivations of left faithful rings, Comm. Algebra 27 (1999), no. 8, 4057-4073. 
[12] _ Semiprime rings with differential identities, Bull. Inst. Math. Acad. Sinica 20 (1992), no. 1, 27-38.

[13] W. S. Martindale III, Prime rings satisfying a generalized polynomial identity, J. Algebra 12 (1969), 176-584.

[14] M. A. Quadri, M. S. Khan, and N. Rehman, Generalized derivations and commutativity of prime rings, Indian J. Pure Appl. Math. 34 (2003), no. 9, 1393-1396.

[15] B. Zalar, On centralizers of semiprime rings, Comment. Math. Univ. Carolin. 32 (1991), no. $4,609-614$

VincenZo De FiLipPis

DI.S.I.A., FACUlty of Engineering

University of Messina, 98166, Messina, Italy

E-mail address: defilippis@unime.it

Shuliang Huang

Department of Mathematics

Chuzhou University

Chuzhou Anhui, 239012, P. R. China

E-mail address: shulianghuang@sina.com 